\title{
The Analysis of Left Ventricular Ejection Fraction after Minimally Invasive Surgery for Primary Mitral Valve Regurgitation
}

\author{
Yukiharu Sugimura ${ }^{1}$, Shintaro Katahira ${ }^{1}$, Philipp Rellecke ${ }^{2}$, Hiroyuki Kamiya ${ }^{3}$, Jan-Philipp \\ Minol $^{1}$, Moritz Immohr ${ }^{4}$, Hug Aubin ${ }^{1}$, Stephan Sixt ${ }^{1}$, Patrick Horn ${ }^{1}$, Ralf Westenfeld ${ }^{1}$, \\ Torsten Doenst ${ }^{5}$, Artur Lichtenberg ${ }^{1}$, and Payam Akhyari ${ }^{1}$ \\ ${ }^{1}$ University Hospital Dusseldorf \\ ${ }^{2}$ Affiliation not available \\ ${ }^{3}$ Asahikawa Medical University \\ ${ }^{4}$ Heinrich Heine University Düsseldorf \\ ${ }^{5}$ Friedrich Schiller University Jena
}

September 11, 2020

\begin{abstract}
Background: Although minimally invasive mitral valve surgery (MIMVS) has become the first choice for primary mitral regurgitation (MR) in recent years, clinical evidence in this field is yet limited. The main focus of this study was the analysis of preoperative (Pre), postoperative (Post) and 1-year follow-up (Fu) data in our series of MIMVS in order to identify factors that have an impact on the left ventricular ejection fraction (LVEF) evolution after MIMVS. Methods: We reviewed the perioperative and 1-year follow-up data from 436 patients with primary MR (338 isolated MIMVS und 98 MIMVS combined with tricuspid valve repair) to analyzed patients baseline characteristics, the change of LV size, the postoperative evolution of LVEF and its factors, and the clinical outcomes. Results: The overall mean value of EF slightly decreased at 1-year follow-up (mean change of LVEF: $-2.63 \pm 9.00 \%$ ). A significant correlation was observed for PreEF und EF evolution, the higher PreEF the more pronounced decreased EF evolution (in all 436 patients; $\mathrm{r}=-0.54, \mathrm{p}<0.001$, in isolated MIMVS; $\mathrm{r}=-0.54, \mathrm{p}<0.001$, in combined MIMVS; $\mathrm{r}=-0.53, \mathrm{p}<0.001)$. Statistically significant differences for negative EF evolution were evident in patients with mild or greater tricuspid valve regurgitation (TR) (in all patients; $\mathrm{p}<0.05, \mathrm{OR}=1.64$, in isolated MIMVS; $\mathrm{p}<0.01, \mathrm{OR}=1.93$, respectively). Overall clinical outcome in NYHA classification at 1 year was remarkably improved. Conclusions: Our results suggest an excellent clinical outcome at 1 year, although mean LVEF slightly declined over time. TR could be a predictor of worsened FuEF in patients undergoing MIMVS.
\end{abstract}

\section{Hosted file}

MICMitral-EF_02.09.2020 . docx available at https://authorea.com/users/355890/articles/480575the-analysis-of-left-ventricular-ejection-fraction-after-minimally-invasive-surgery-forprimary-mitral-valve-regurgitation 


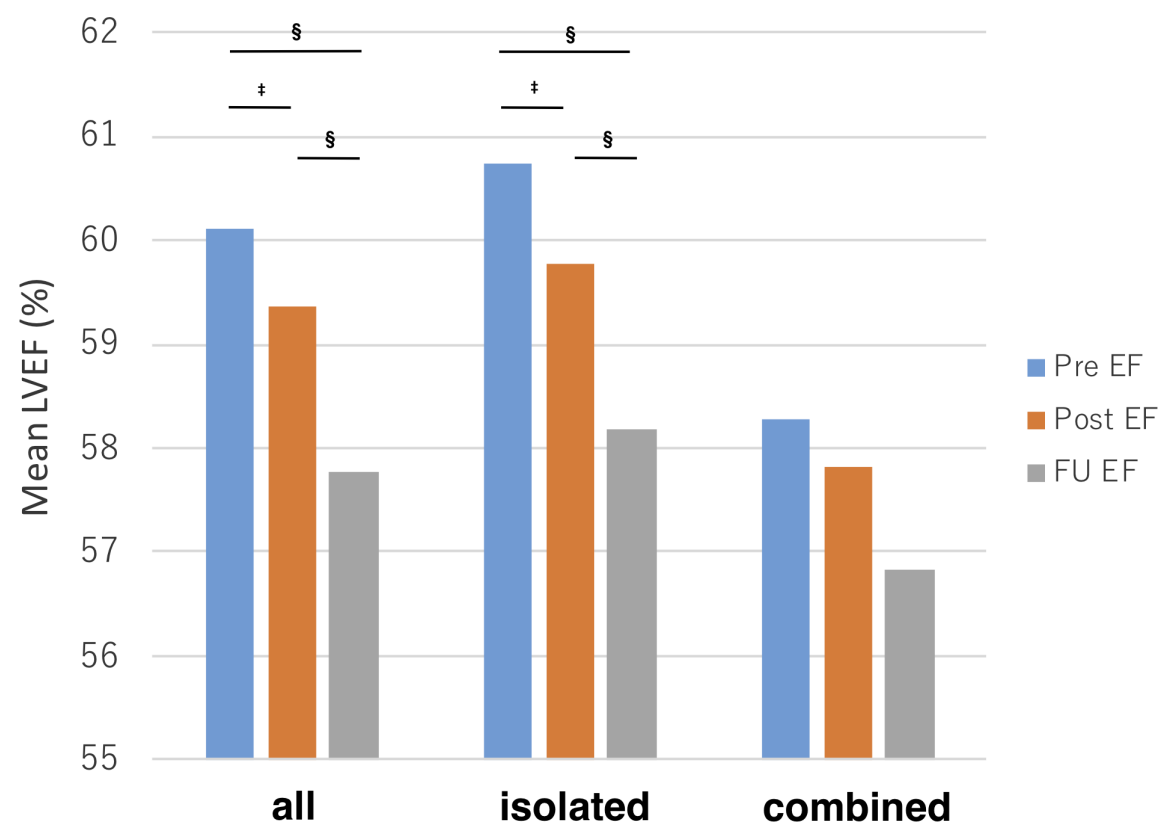

A

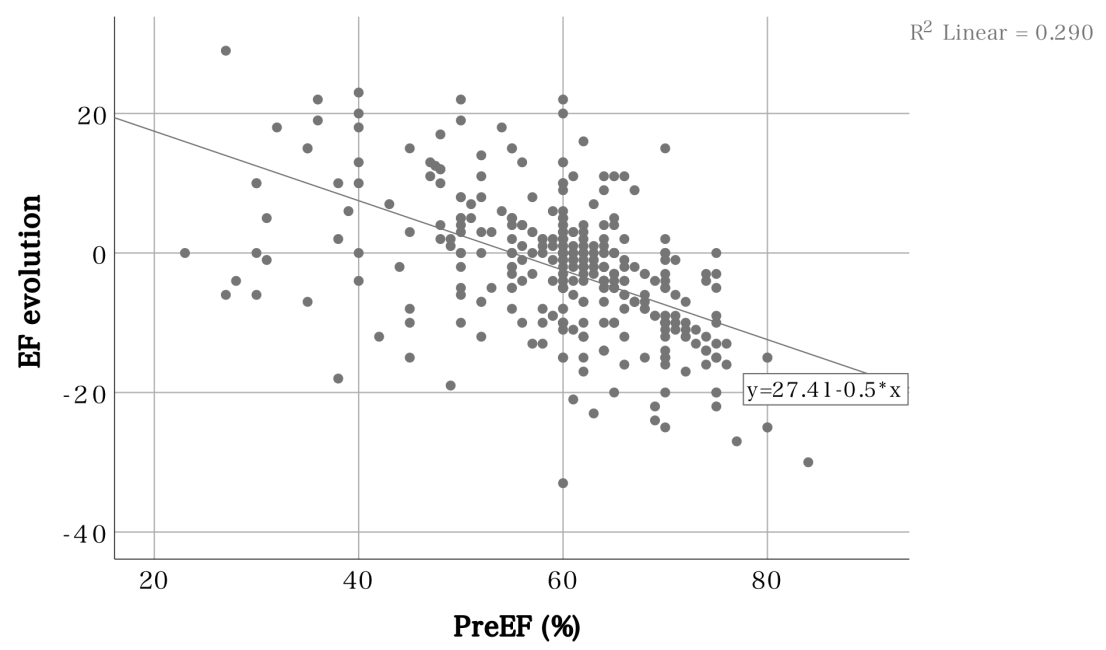


B

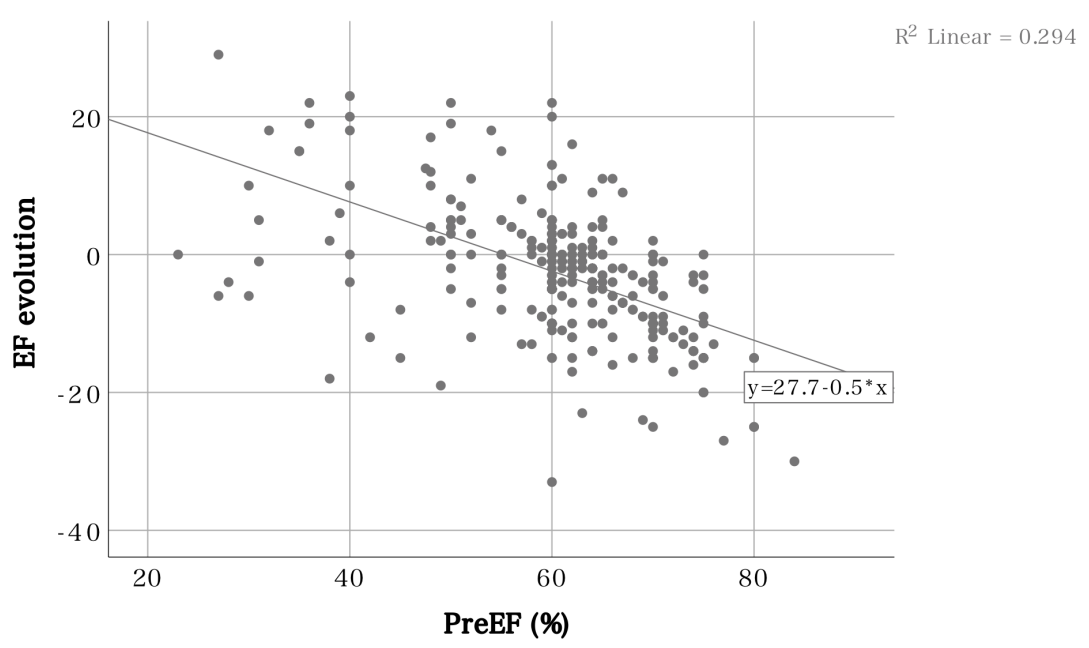

C

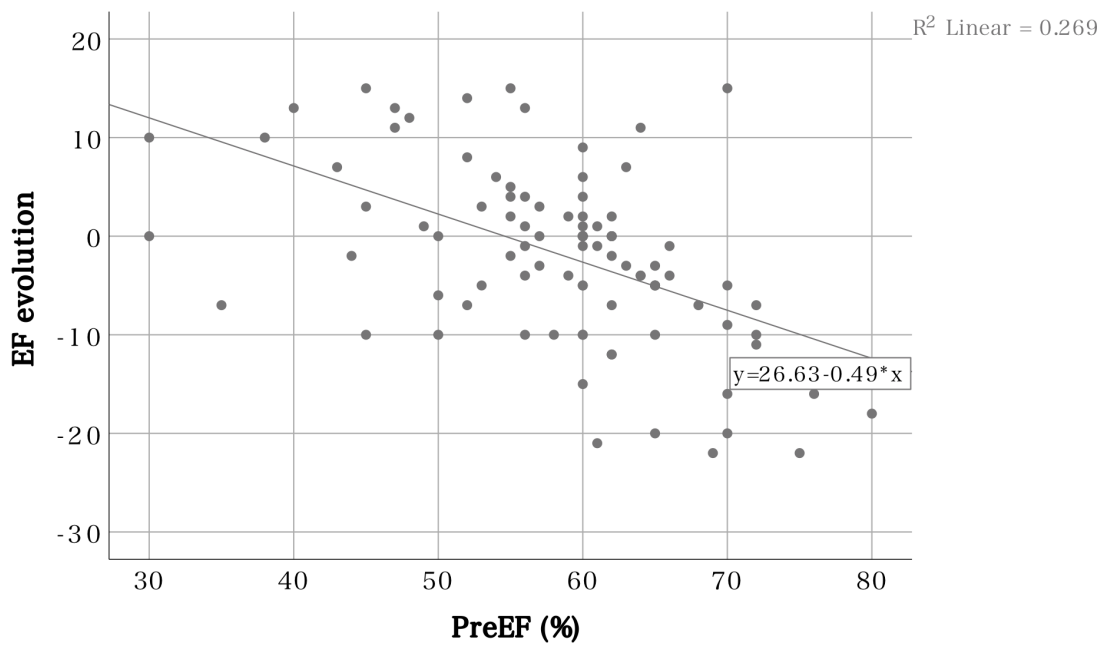




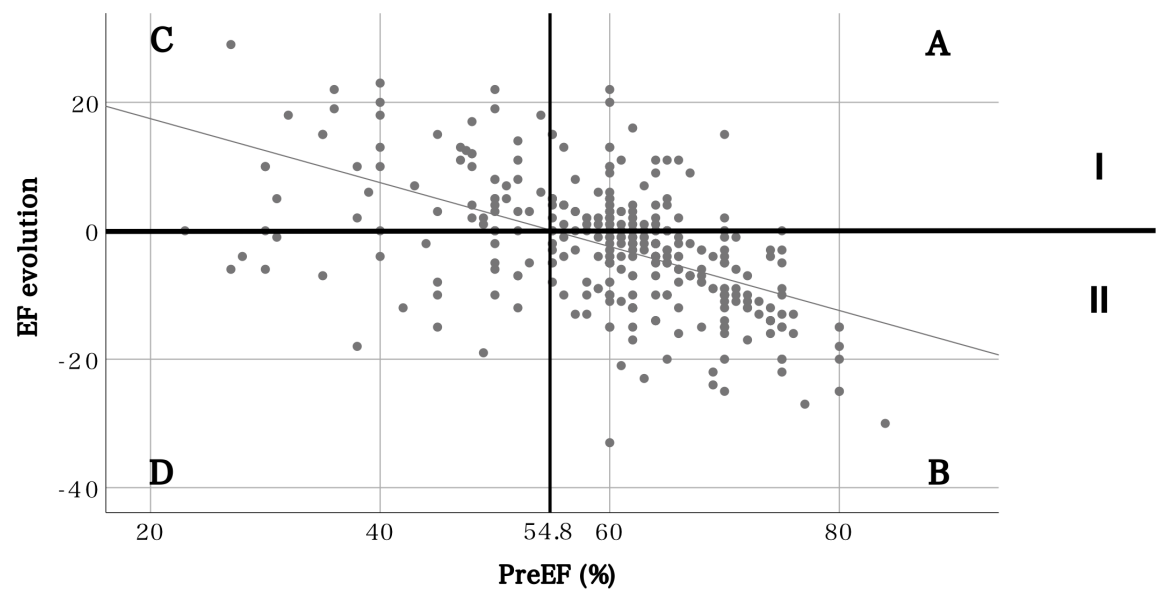

Seite 1 


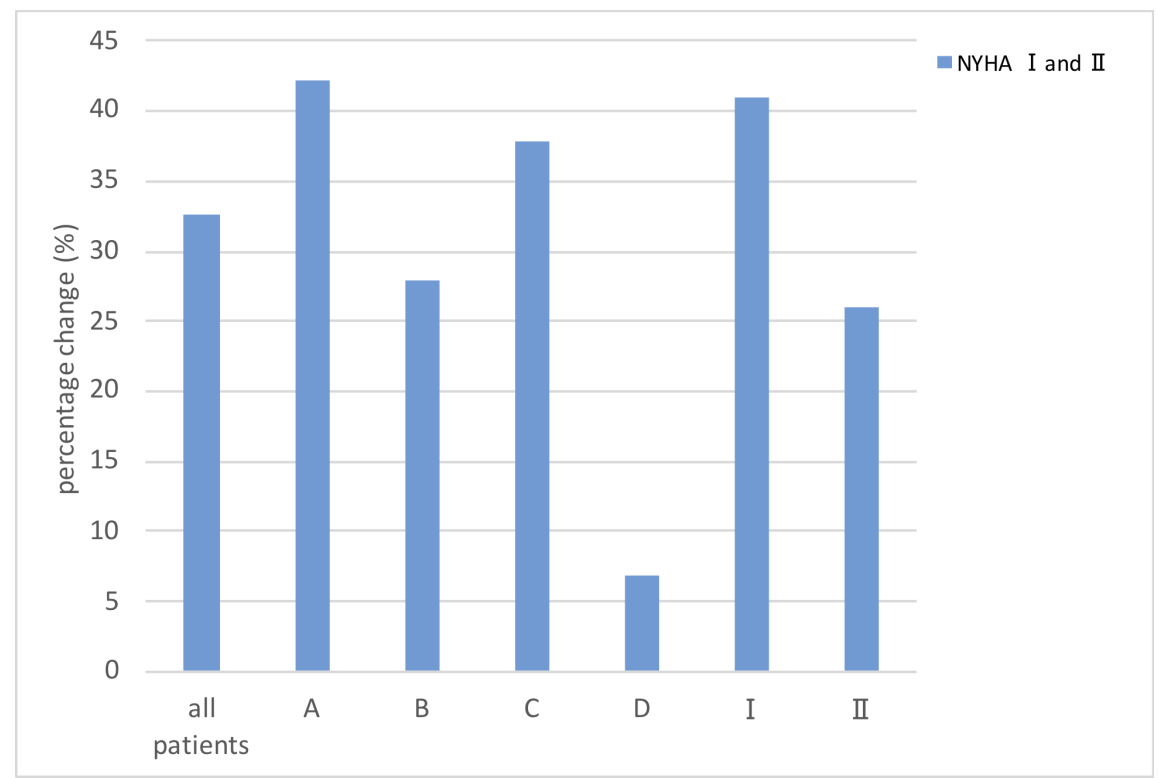

